\title{
Characteristics Analysis at Prefix Granularity: A Case Study in an IPv6 Network
}

\author{
Fuliang $\mathrm{Li}^{1}$, Jiahai Yang ${ }^{2}$, Xingwei Wang ${ }^{1 *}$, Tian $\operatorname{Pan}^{2}$, Changqing $\mathrm{An}^{2}$, Jianping $\mathrm{Wu}^{2}$
}

\begin{abstract}
As IPv6 has much larger address space than IPv4, investigating the characteristics of IPv6 prefixes is of great benefit to understand and manage IPv6 networks. In this paper, 1) we conduct a case study on prefix-level characteristics in IPv6 world. We find that the number of assigned prefixes and the coverage areas are increasing and expanding. Traffic and packet distribution across prefixes are highly skewed. The sizes of active prefixes are relatively stable over time. These results provide experimental basis for routing cache design in IPv6 world. If we update the routing cache once an hour, we only need to reserve 5184 bytes memory for the worst-case burst changes. 2) In addition, we find that $61 \%$ destination prefixes have more than two paths to reach, which shows that multihoming path diversity of the studied IPv6 network is obvious. Therefore, we propose an experimental framework with the ability of measuring the performance of each destination prefix, tuning the path for each destination prefix with the best performance, and evaluating the effect during and after the performance tuning. IPv6 has been experiencing fast development as the next generation network. Hence understanding prefix-level characteristics and enhancing performance management are essential for IPv6 networks.
\end{abstract}

Keywords - network management; IPv6; prefix-level; characteristics; performance;

\section{Introduction}

Due to the exhaustion of IPv4 addresses, transition to IPv6 (Internet Protocol-IP version 6) [1-3] is imminent and challenging. As IPv6 has much larger address space than IPv4, unveiling prefix-level characteristics can offer deep insights into understanding and management of IPv6 networks. A Prefix is used to identify a subnet or even a network. It is composed of an address, a slash character (/) and the bit-length of the prefix, e.g., 166.111.1.0/24 and 2001:da8::/32. In this paper, we investigate prefix-level characteristics (such as assignment, activity, and traffic distribution, etc.) in IPv6 world motivated by the following two aspects.

(1) High-speed packet forwarding capability in router's data plane is unquestionably the cornerstone of today's blooming Internet. To guarantee the line-rate packet forwarding in the face of inflated network traffic, specific-designed hardware is needed. E.g., TCAM (Ternary Content Addressable Memory) chips are conventionally embedded into router's data plane to speed up the packet processing, achieving one lookup operation each clock cycle on average [4]. However, except for the high performance, TCAM is also expensive as well as power-hungry. Provided that the backbone routing table undergoes an explosive growth these days (with the routing table entries approaching $500 \mathrm{~K}$ in year 2013 [5]), it is not a cost-effective solution to squeeze the entire routing table into the expensive TCAM hardware. Fortunately, routing prefix exhibits power-law behaviors that only a small portion of prefixes will be heavily accessed in a period of time [6], which suggests introducing a routing cache of popular prefixes can be a great performance enhancement to the packet forwarding. In this way, the heavy-hitters can be stored temporarily in fastspeed, limited-capacity TCAM chip, leaving others in the secondary storage such as DRAM (Dynamic Random Access Memory), maintained under the rule of the cache replace algorithm. The caching performance heavily relies on the traffic characteristics, such as traffic distribution across routing prefixes. While the traffic distribution across prefixes is widely studied in regard of IPv4 networks [7-10], to the best of our knowledge, there are few works concerning IPv6 networks. Therefore, analyzing prefix-level characteristics, especially traffic distribution across prefixes in IPv6 world is needed.

(2) IPv6 is in its initial but fast development phase [38], so abundant peering relationships among IPv6 networks are expected. That is to say, for a packet going to a destination prefix, there may be more than one path (next hop). Give this, multihoming (i.e., a customer or ISP (Internet Service Provider) network connecting to multiple peers) is needed to be understood from the perspective of an operational IPv6 network. In addition, due to the demand of some real-time services (such as online games, financial transactions, video conferences, etc.), guaranteeing the network with a good performance is significant. Performance tuning at prefix granularity can be conducted based on multihoming path diversity characteristics. Multihoming analysis and optimization [27-32] are widely studied in these years. However, the following three problems deriving from the engineering practice may still exist and should be considered together. First of all, information gathered from the network is limited. Many kinds of information can be collected, such as traffic, RIB (Routing Information Base), configuration files, delay, packet loss, link capacity, etc., to provide a solid basis for performance tuning. Thus, multi-source measurements are necessary.

*Corresponding author: Xingwei Wang, Room 1205, Main Building, Northeastern University, Shenyang 110819, China; Email Address, wangxw@mail.neu.edu.cn, Phone, 0086-24-83687575; Fax, 0086-24-83687575 
Secondly, any network adjustments are finally realized through device-level configuration. Massive and repetitive configuration tasks lay heavy burden on operators [33-34], so simple but practical methods are required to support automatic performance tuning. Thirdly, effect (i.e., instability and effectiveness) caused by adjustments is less unknown and fed back to instruct performance tuning. On the one hand, during the performance tuning, instability like routing oscillation, packet disordering and missing, packet delay increasing, etc., may be brought in [35-37]. We should quantify these dynamics and offer positive advice on how to reduce the instability caused by network adjustments. On the other hand, effectiveness of adjustments, i.e., whether we gain the satisfying performance profits after the performance tuning is also needed to be further checked. Results of effectiveness evaluation can be used to maximize the performance profits through repeating adjustments.

In this paper, we conduct a case study on prefix-granularity characteristics in IPv6 world, which could provide good insights for routing cache design and network performance management. Our main findings and contributions are summarized as follows. (1) We analyze the prefix-level characteristics based on the $\operatorname{RIR}^{1}$ (Regional Internet Registry) data and the traces (i.e., network traffic) of an IPv6 network. We find that more prefixes are assigned in length of 32, while more prefixes are active in length of 48. However, most assigned prefixes ${ }^{2}$ and active prefixes ${ }^{3}$ (length) are within the closed interval of [32, 48]. Distributions of packet and traffic are highly skewed across prefixes. The size of active prefixes is relatively stable over time. Only 5184 bytes memory needs to be reserved for the worst-case burst changes if the update frequency is one hour. (2) In addition, we analyze the multihoming path diversity of IPv6 prefixes and put forward a performance management framework that integrates network information Monitoring, performance Tuning and effect Evaluation (so-called MTE) together. MTE can measure the performance of each destination prefix with multiple upstream paths, tune the path for each destination prefix with the best performance, and evaluate the effect during and after the performance tuning. We validate MTE based on a typical scenario in the real network environment and the evaluation results reveal the practicality of MTE.

The remainder of this paper is organized as follows. The related works are presented in Section 2. Section 3 shows our analysis results of prefix-level characteristics, as well as insights into routing cache design of an IPv6 network. Then multihoming path diversity of the studied IPv6 network and a framework integrating information monitoring, performance tuning and effect evaluation are described in Section 4. Section 5 presents implications of our analysis and validation results. Conclusions and future remarks are discussed in Section 6.

\section{Related Work}

Related works are presented from two aspects. We begin with discussion of existing works on data analysis to understand the development and usage of IPv6 networks. Then, we show the studies that have been conducted on routing cache design and performance optimization based on prefix-level characteristics.

\subsection{Studies on IPv6 development and usage}

Various kinds of data are collected and analyzed, including RIR logs, BGP (Border Gateway Protocol) dumps, DNS (Domain Name Server), WEB and FTP (File Transfer Protocol) server logs, traceroute data, netflow records, packet level datasets, web access data [11-20]. Colitti et al. [11] measured IPv6 adoption from the perspective of a website operator. Malone [12] analyzed transition technologies based on the data collected from FTP and DNS servers. Karpilovsky et al. [13] found that although allocations of IPv6 addresses are growing, many prefixes are used either long time after allocation or not used at all. P. Hicks [14] found that the IPv6 AS-core map is comprised of 715 AS nodes in 2010 compared with 515 nodes in 2009. Investigations of netflow records and packet level datasets indicated that IPv6 development in China is progressing rapidly [15-16]. Analysis of IPv6 traffic was performed to reveal what happened after the World IPv6 Day [17-18]. Nikkah et al. [19] found that if AS-level paths are the same, IPv6 performance is similar to IPv4 performance. Dhamdhere et al. [20] discovered that IPv6 deployment is strong in core, lagging at edge. Berger et al [49] characterized the inter-relation of IPv4 and IPv6 among Internet DNS resolvers by deploying both active and passive measurement techniques. Czyz et al. [50] collected and analyzed unclaimed traffic on the IPv6 Internet by announcing five large /12 covering prefixes. Luckie, Beverly and et al. [51-52] devoted to alias resolution technique for IPv6, which represents a step toward understanding the Internet's IPv6 router-level topology. Lutu et al. [53] found that the fraction of limited visibility address space (i.e., not present in all the global routing tables of the operational networks.) is similar in the IPv4 and IPv6 Internet. Alzoubi et al. [54] found no evidence of performance penalty for unilateral enabling of IPv6,

\footnotetext{
${ }^{1}$ RIR is an organization that manages the allocation and registration of IP addresses and autonomous system numbers. RIR divides the world into five RIRs, including African Network Information Center (AFRINIC), American Registry for Internet Numbers (ARIN), Asia-Pacific Network Information Centre (APNIC), Latin America and Caribbean Network Information Centre (LACNIC), Réseaux IP Européens Network Coordination Centre (RIPENCC).

${ }^{2}$ Assigned prefixes refer to the prefixes that are allocated to institutions, organizations, etc., by RIRs.

${ }^{3}$ Active prefixes refer to the assigned prefixes that are being used in the Internet.
} 
which provides basis for sites when they consider their IPv6 migration strategy. Czyz et al. [55] provided a holistic view and draw an updated picture of the current state of IPv6 adoption from the aspects of addressing, naming, routing, end-to-end reachability, traffic and performance. Recently, V. Giotsas et al. [56] adapted the CAIDA's (Center for Applied Internet Data Analysis) IPv4 AS relationship inference algorithm [57] to accurately infer IPv6 AS relationships, which could model and understand IPv6 AS topology evolution. R. Beverly et al. [58] proposed an active measurement method to measure and characterize IPv6 router availability. They found that core routers tend to have longer uptimes than border routers. R. Padmanabhan et al. [59] put forward a new alias resolution technique for IPv6 using unused addresses. R. Beverly et al. [60] investigated whether candidate IPv4 and IPv6 addresses belong to the same machine through an active measurement technique. D. Plonka et al. [62] developed a methodology to classify IPv6 addresses. They used the temporal analysis technique to determine prefix and address stability over time and employed the spatial analysis technique to determine the structure in which prefixes and addresses are contained. Many aspects of IPv6 network have been investigated across wide range of datasets and measurement methods, but few studies characterize IPv6 networks at prefix granularity deeply. In this paper, we conduct a case but relatively comprehensive study on IPv6 prefix-level characteristics, including prefix assignment, prefix activity, traffic distribution across prefixes, changes of active prefixes, etc., which can make a positive contribution to helping us fully understand IPv6 networks and provide an experimental basis for routing cache design and performance optimization in IPv6 networks..

\subsection{Studies based on prefix-level characteristics}

Prefix-level characteristics analysis has the following two benefits. On the one hand, network traffic exhibits remarkable temporal locality in the Layer-3 in IPv4 world, as observed by Thompson et al. [21]. To exploit such locality to improve the processing performance in packet lookup as well as lower down the TCAM cost, various schemes introduce routing cache in highspeed routers [22-26]. There are mainly two concerns in these schemes. The first is cache granularity. Conventionally, they put IP addresses into the routing cache. However, to reduce the cache size, prefixes are filled into the cache entries [22]. The second is the way to prevent cache-hiding problem since the IP address lookup must satisfy longest prefix match. The overlapped prefixes are eliminated via leaf-pushing to meet satisfaction [24]. While all above work utilizes the traffic locality and uneven distribution across prefixes in IPv4 world, we need the similar measurement in IPv6 world. R. Guo [61] mentioned a little that the traffic trace exits temporal locality of in view of the number of unique IPv4 and IPv6 addresses. However, they concentrated on routing table compaction scheme design based on TCAM rather than prefix-level characteristics analysis. The most relevant work to this paper has shown the analysis of traffic distribution across prefixes and evaluation on routing caching performance based on the traces of an IPv4 network [10]. In this paper, we conduct some similar analyses mainly based on the datasets of an IPv6 network [39], which is already a production network, so our datasets are convincing to explore prefix-level characteristics of IPv6 networks. And our analysis results, which we believe, can provide an experimental basis for routing caching or router design in IPv6 world.

On the other hand, as applications such as video conference, IPTV, VoIP and online games become prevalent, keeping network availability as well as good performance is crucial. To guarantee high bandwidth and low delay, as well as enhance the reliability in the event of a failure in a peering network, a customer or ISP network with multiple upstream connections (i.e., multihoming) is encouraged in ISP networks. A. Akella et al. [27] proposed performance and reliability metrics to quantify the benefits from multihoming in IPv4 networks. They found that multihoming could improve performance if selecting the right set of providers. A. Akella et al. [28] also conducted a comparison of overlay routing and multihoming route control. They found that multihoming route control can offer performance similar to overlay routing. D. K. Goldenberg et al. [29] took advantage of multihoming and designed a series of algorithms to optimize cost and performance for multihomed users. Multihoming also has been introduced into content distribution networks (CDNs). H. H. Liu et al. [30] optimized both performance and cost for content multihoming, i.e., content publishing using multiple CDNs. J. M. Wang et al. [31] realized an alternative content multihoming approach through choosing an authoritative CDN to handle the potential performance trap of individual CDN. C. De Launois et al. [32] used multiple provider-dependent aggregatable (PA) prefixes to realize IPv6 multihoming, which could enhance Internet path diversity and further benefit from low delays. Different from previous works, we investigate the multihoming of a production IPv6 network. More importantly, in view of the multihoming path diversity and due to the practical operation requirements of the studied IPv6 network, we adopt a centralized mechanism to optimize the performance for each destination prefix with multiple external links (either to a single ISP, or to different providers). It is the first approach that integrates high-level effect evaluating, middle-level automatic performance tuning and low-level network information monitoring. This is different from the prevalent term of software-defined networking (SDN), which typically requires switches to support a new application programming interface, like OpenFlow. We do not require any special support from the routers, so it is easy to deploy the mechanism without extra costs for devices.

\section{Prefix-level Characteristics Analysis}

In this section, we analyze the prefix-related characteristics based on the traces of an IPv6 network, as well as present the assignment and activity of IPv6 prefixes. More importantly, we show the insights on IPv6 routing case design according to the prefix-level characteristics analysis. 


\subsection{Assignment and Activity of IPv6 Prefixes}

Assignment and activity of IPv6 prefixes are less understood over time. However, these kinds of information can show prefix distribution in length, which influences the decision on routing cache model. For example, if nearly all active prefixes are $/ 48$, then we can adopt "flat", uni-cast caching [10]. E.g., in IPv4 world, most prefixes are at the length of /24. Such characteristic is utilized by many routing search algorithms to save hardware expense opportunistically when implementing longest prefix match. However, if active prefix distribution in length is dispersed, the uni-cast caching is no longer applicable. We fill the gap of IPv6 world from many aspects.

\subsubsection{Prefix assignment among RIRs}

RIRs data is significant for understanding how IPv6 prefixes are allocated. As depicted in Table 1, IPv6 prefixes allocated to the five RIRs (APNIC, ARIN, RIPENCC, AFRINIC and LACNIC) present obvious growth during the past three years. In 2011, RIPENCC was allocated 4205 prefixes. While in 2013, the number of prefixes allocated to RIPENCC is 7365 , which shows a $75 \%$ increase. The number of prefixes allocated to LACNIC varies from 275 in 2011 to 1790 in 2013, which presents the largest growth rate of 551\%. We also find that the number of areas (i.e., countries and regions) grows from 193 in 2011 to 228 in 2013. Overall, the high growth rates of prefixes and the more comprehensive coverage areas imply that IPv6 is in a fast development phrase.

Table 1. Prefix assignment among RIRs

\begin{tabular}{c|c|c|c|c}
\hline \multirow{2}{*}{ TYPE } & \multicolumn{2}{|c|}{ Number of Areas } & \multicolumn{2}{c}{ Number of Prefixes } \\
\cline { 2 - 5 } & $\mathbf{2 0 1 1 / 1 0}$ & $\mathbf{2 0 1 3 / 7}$ & $\mathbf{2 0 1 1 / 1 0}$ & $\mathbf{2 0 1 3 / 7}$ \\
\hline RIPENCC & 73 & 80 & 4205 & 7365 \\
\hline APNIC & 47 & 53 & 2037 & 3024 \\
\hline ARIN & 12 & 17 & 2542 & 3508 \\
\hline LACNIC & 24 & 30 & 275 & 1790 \\
\hline AFRINIC & 37 & 48 & 227 & 359 \\
\hline Total & 193 & 228 & 9286 & 16046 \\
\hline
\end{tabular}

\subsubsection{Prefix assignment in length}

The length of the assigned prefixes is heuristic for effective routing cache design. As shown in Table 2, the number of the prefixes, whose lengths are less than 32 , presents the most obvious growth rate of $551 \%$. The number of $/ 32$ prefixes varies from 6788 in 2011 to 10790 in 2013, showing a 59\% increase, which is lower than the 80\% increase of /48 prefixes from 1825 to 3281. In addition, we also find that prefixes of $/ 32$ and $/ 48$ dominate the assigned prefixes. Prefixes of $/ 32$ account for $71 \%$ and $67.2 \%$ of the total assigned prefixes in 2011 and 2013 respectively, while the ratios are $19.1 \%$ and $20.4 \%$ for prefixes of $/ 48$. Although prefixes of $/ 32$ are assigned much more than $/ 48$, the proportion of prefix of $/ 32$ is falling, while for prefixes of $/ 48$, the proportion is increasing. Although 128-bit is allocated in packet header under IPv6 specification, the width of routing cache entries in IPv6 world can be much shorter, only holding popular prefixes. 48-bit width TCAM entry will be sufficient to cache a majority of traffic. A more greedy strategy is to use both 32-bit and 48-bit cache entries to correspond to the "double-peak" characteristics in IPv6 prefixes, resulting in an even smaller routing cache memory footprint. The packets miss the prefixes whose length is equal to or smaller than 48-bit will be sent to the backup routing table kept in a secondary storage, which will not incur a performance reduction on average due to the small amount. If software-based routing table design is chosen to reduce the system budget, the 3248 (prefixes in the closed interval of $[32,48]$ ) heuristic could make an aid as well. The trie data structure can be tuned to allocate 32 or 48 bit for one step length to search the most popular prefixes in one memory access. This saves multiple memory access times when using traditional binary trie structure to represent a routing table.

Table 2. Assigned Prefix distribution in Length (RIRs)

\begin{tabular}{c|c|c|c|c|c}
\hline TYPE & $\mathbf{3 3 2}$ & $\mathbf{3 2}$ & $\mathbf{( 3 2 , 4 8}$ & $\mathbf{4 8}$ & $>$ \\
\hline $\mathbf{2 0 1 1} / \mathbf{1 0}$ & 169 & 6788 & 499 & 1825 & 3 \\
\hline $\mathbf{2 0 1 3 / 0 7}$ & 1101 & 10790 & 869 & 3281 & 3 \\
\hline growth rate & $551 \%$ & $59 \%$ & $74 \%$ & $80 \%$ & $0 \%$ \\
\hline
\end{tabular}

\subsubsection{Prefixes activity observed from RIBs}

We gather RIBs from two observation points - CERNET2 [39] on Nov 15, 2013 and University of Oregon route views project [47] on Nov 20, 2013. As shown in Table 3, there are a total of 14888 active prefixes configured on the border routers of CERNET2 and the number of active prefixes calculated for route view is 16229. Active prefixes of the two observation points are approximately in the same level. We also find that prefixes of $/ 32$ and $/ 48$ are most commonly used. And there are more active prefixes of /48 than prefixes of /32, which is different from the result shown in Table 2, where more prefixes of $/ 32$ are assigned. However, active prefixes in the closed interval of $[32,48]$ also account for most of the active prefixes $(96.5 \%$ and $93.1 \%$ of the two 
observation points respectively). Hence, the routing cache design thoughts stated above are appropriate for the results observed from RIBs as well.

Table 3. Active prefix distribution in Length (RIBs)

\begin{tabular}{c|c|c|c|c|c|c}
\hline TYPE & $<32$ & $\mathbf{3 2}$ & $\mathbf{( 3 2 , 4 8 )}$ & $\mathbf{4 8}$ & $\mathbf{\text { total }}$ \\
\hline CERNET2 & 510 & 5298 & 3065 & 6011 & 4 \\
\hline Route view & 521 & 5402 & 3253 & 6452 & 14888 \\
\hline
\end{tabular}

\subsubsection{Prefixes activity observed from UPDs}

The UPDs (update messages) are collected from the border routers of CERNET2. We use one-week scale data to illustrate prefixes activity observed from UPDs. As shown in Table 4, we gather a total of 28726343 update messages during the observation period. That is to say, there are 4103763 UPDs/day (47 UPDs/s) and 1929 UPDs/prefix. Further analyses show that during the one-week observation, only 8356 prefixes $(56.13 \%$ of the total prefixes) contribute UPDs. In addition, top $20 \%$ of the prefixes account for $60 \%$ of the UDPs. We also find that prefixes whose lengths are within the range of [32, 48] contribute 96.5\% of the total update messages. The proportion is similar to the result observed from RIBs. We also capture the withdraw messages and the results show that the number of withdraw messages is far less than the number of update messages. Prefixes in the closed interval of $[32,48]$ account for $97 \%$ of the total withdraw messages. The larger number of update messages and the less of withdraw messages reveal that message of IPv6 prefixes are active but lifecycles (i.e., active periods of the prefixes) of them are relatively stable. On average, 47 prefix updates per second is fairly tolerable for contemporary hardware operation on the routing cache; however, the hardware still needs to be over provisioned in case of the arrival of burst updates.

Table 4. Active prefix distribution in Length (UPDs)

\begin{tabular}{c|c|c|c|c|c|c}
\hline TYPE & $\mathbf{3 2}$ & $\mathbf{3 2}$ & $\mathbf{( 3 2 , 4 8 )}$ & $\mathbf{4 8}$ & $\mathbf{\text { 48 }}$ & $\mathbf{\text { total }}$ \\
\hline Update & 988531 & 10108761 & 5980316 & 11641154 & 7581 & 28726343 \\
\hline Withdraw & 163 & 1082 & 1433 & 4863 & 12 & 7608 \\
\hline
\end{tabular}

\subsection{Prefix-related characteristics of IPv6 traces}

Prefix-related characteristics of IPv6 traces can also provide essential basis for routing cache design. For example, traffic distribution across prefixes can show which prefixes contribute most traffic. Prefix distribution across different time scales can tell us how large a routing cache is needed. Hence, we investigate many prefix-related characteristics according to the traces of CERNET2 in this section.

CERNET2 [39] (The next generation China Education and Research Network) is a nation-wide pure IPv6 backbone network. As depicted in Fig. 1, CERNET2 interconnects to domestic CNGI backbones and international research networks through the exchange point of CNGI-6IX. There are two main IPv6 traces - DS1 and DS2. DS1 is gathered from 2011-10-24 00:00 to 2011-1025 23:59 (5.4 TB). DS2 is gathered from 2013-7-17 14:00 to 2013-7-17 14:59 (193 GB). In this paper, we use a sub-data (hourscale) of DS1 from 2011-10-25 14:00 to 2011-10-25 14:59 to make a comparison to DS2. In 2011, the traffic volume value stays slightly higher or lower than $4 \mathrm{Gbit} / \mathrm{s}$. However, traffic of the hour-scale data in 2013 varies dramatically. The value is about 7.7 Gbit/s at peak time and about $5 \mathrm{Gbit} / \mathrm{s}$ in the trough. The results illustrate that the traffic presents an obvious increase during the past two years. In addition, we can see that CERNET2 is heavily used, and our dataset is convincing to explore prefix-related characteristics of IPv6 traces.

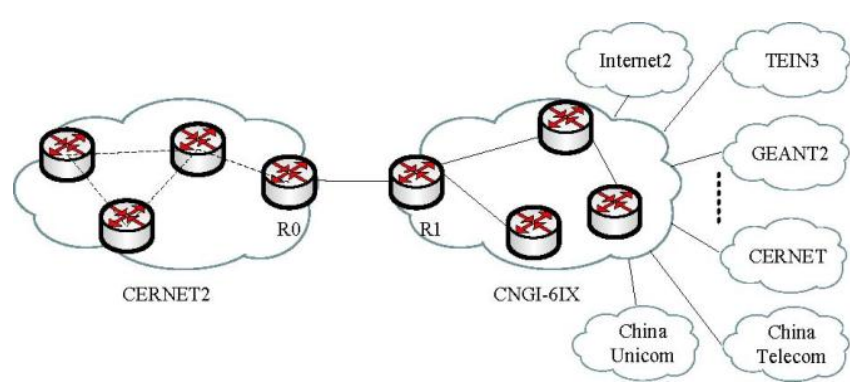

Fig. 1. Topology of CERNET2 and CNGI-6IX

\subsubsection{Prefix activity observed from traffic}


We have known that many prefixes have been allocated, but whether they are in actual use and generating IPv6 traffic is unknown. So according to the traces of CERNET2, we investigate the activity of prefixes from the aspect of passive measurement (in views of communications with CERNET2). As depicted in Table 5, 3222 active prefixes (accounting for 34.8\% of the total assigned prefixes) can be observed in the whole day of 2011-10-24. When we conduct the same analysis based on the one-hour scale dataset, we find that the numbers of the active prefixes are 1700 (18.3\%) in 2011 and 2406 (15\%) in 2013 respectively. The results reveal that if we design a routing cache for the border routers of CERNET2, the size of routing cache is easy to control for a remarkably acceptable cache hit rate. E.g., we only need to cache $15 \%$ of the total prefixes to safely keep a very high cache hit rate for one-hour duration. We also analyze the active prefixes distributed in five RIRs according to the one-hour scale dataset. As shown in Table 6, the number of active prefixes presents obvious growth during the past two years. APNIC, ARIN and RIPENCC present the similar growth rates, while growth rates of LACNIC and AFRINIC are $176.7 \%$ and $100 \%$ respectively. As the widely deployment of IPv6 networks, the active prefix number is growing fast, which potentially indicates a larger capacity routing cache.

Table 5. Number of active prefixes observed from traffic

\begin{tabular}{c|c|c|c}
\hline TYPE & $\mathbf{2 0 1 1 / 1 0 / 2 4}$ & $\mathbf{2 0 1 1 / 1 0 / 2 5}$ & $\mathbf{2 0 1 3} / 7 / \mathbf{1 7}$ \\
& $\mathbf{0 0 : 0 0 - 2 3 : 5 9}$ & $1700-14: 59$ & $\mathbf{1 4 : 0 0 - 1 4 : 5 9}$ \\
\hline Number & 3222 & $18.3 \%$ & 2406 \\
\hline Proportion & $34.8 \%$ & $15 \%$ & \\
\hline
\end{tabular}

Table 6. Number of active prefix distributed in five RIRs

\begin{tabular}{c|c|c|c|c|c}
\hline TYPE & APNIC & ARIN & RIPENCC & LACNIC & AFRINIC \\
\hline $\mathbf{2 0 1 0 / 1 0 / 2 5}$ & 356 & 436 & 913 & 30 \\
\hline $\mathbf{2 0 1 3 / 0 7 / 1 7}$ & 469 & 596 & 1228 & 83 \\
\hline growth rate & $31.7 \%$ & $36.7 \%$ & $34.5 \%$ & $17 \%$ \\
\hline
\end{tabular}

\subsubsection{Packet and traffic distribution across prefixes}

Packet and traffic distribution across prefixes are unbalanced. As shown in Fig. 2(a), packet distribution across prefixes is highly skewed. The most popular prefix in 2011 and 2013 is $2001:$ cc0::/32, which accounts for $81.4 \%$ and $61.4 \%$ of the packets respectively. $1 \%$ of the active prefixes account for $98.14 \%$ of the packets in 2011 , and $97.44 \%$ in 2013 . We also investigate traffic distribution across prefixes. As depicted in Fig. 2(b), traffic distribution across prefixes also presents a skewness trend. The most popular prefix in 2011 and 2013 is $2001: 8000:: / 20$, which contributes $86.78 \%$ and $61.72 \%$ of the aggregate traffic respectively. $1 \%$ of the active prefixes account for $98.39 \%$ of the traffic in 2011, while the proportion is $97.75 \%$ in 2013 . Both packets and traffic are highly skewed across prefixes in the studied IPv6 network. The results are strongly supportive for introducing routing cache in IPv6 world. For example, if we keep the top $1 \%$ prefixes in the routing cache stably with the aid of some cache replacement policies, and leave the $99 \%$ less popular prefixes in the backup storage, we will achieve the balance point between cost and performance.

\subsubsection{Relationships among addresses, prefixes and packets}

We have known that packet and traffic distribution are highly skewed across prefixes. However, we want to conduct a further analysis of how many addresses are there and what the packet distribution across addresses is. As shown in Fig. 3(a), address distribution is less skewed across prefixes compared to the results depicted in Fig. $2.1 \%$ of the active prefixes account for $60.6 \%$ and $71.9 \%$ of the addresses in 2011 and 2013 respectively. When the proportion of the active prefixes reaches $20 \%$, the proportion of the active addresses is more than $90 \%$. We also investigate packet distribution across addresses, each of which represents a host or a server outside of CERNET2. As depicted in Fig. 3(b), top 5\% users account for 97.4\% and $99.2 \%$ of the packets in 2011 and in 2013 respectively. According to the results, we can infer that a small part of users contribute most of the packets. The results are similar to the trend of packets distribution across prefixes. In summary, traffic and packets are clustered together in a small part of prefixes and addresses. These results are also useful for routing cache design. For example, in order to accelerate the looking up speed of routing cache, we can inject top 5\% (for the one-hour data of CERNET2, the numbers of top 5\% addresses are 1695 in 2011 and 3870 in 2013) of the addresses as the host routes in the routing cache. 
(a) Packet Distribution

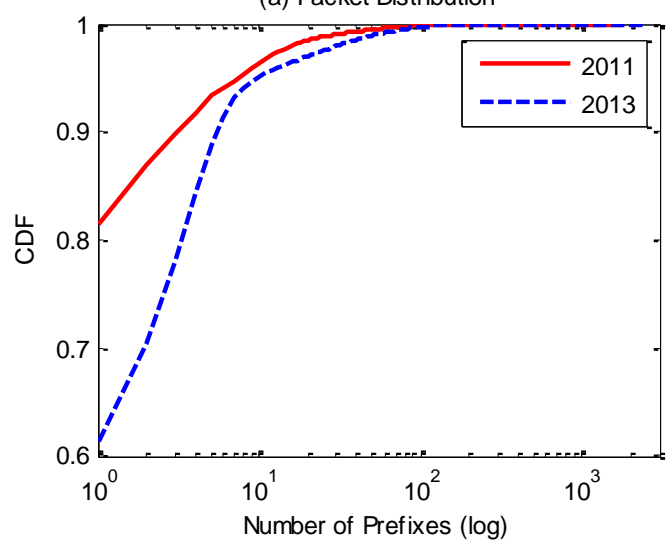

(b) Traffic Distribution

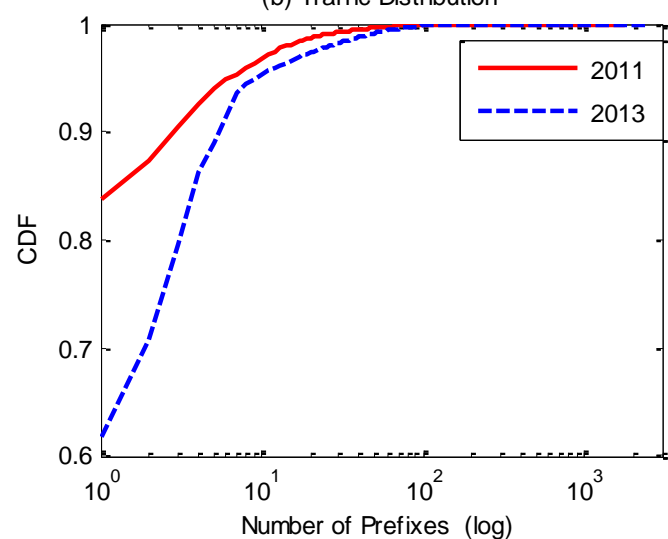

Fig. 2. Packet and traffic distribution across active prefixes
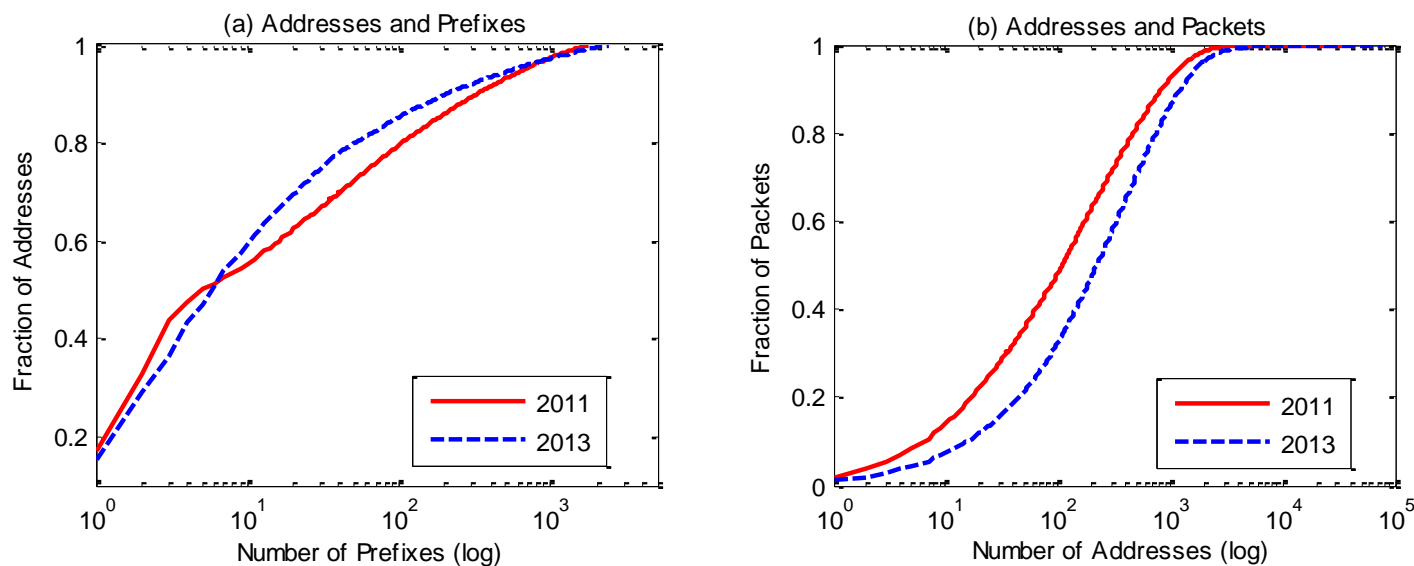

Fig. 3. Relationships among addresses, prefixes and packets

\subsubsection{Prefixes distribution across different timescales}

The active prefix number in a fixed timescale reveals the required cache capacity to hold the prefixes under a given cache miss rate. So the number of the active prefixes over a given period of time and its variation are often used to estimate how large a cache will be needed to achieve a given miss rate [10]. We first make a study on temporal dynamics of traffic destinations according to the one-hour scale dataset. As shown in Fig. 4(a), the number of active prefixes varies from 600 to 700 in 2011, while the number oscillates at 900 in 2013. In the one-minute time interval observation, the standard deviations in the number of active prefixes are 15.7 and 17.3 in 2011 and 2013 respectively. This implies the volume of the traffic flows into the IPv6 tube is growing in recent years with the exhaustion of the IPv4 address pool. We also conduct similar measurement in the view of four different time intervals in a whole day (2011/10/24). As depicted in Fig. 4(b), the number of prefixes varies with a diurnal pattern, i.e., decreasing after the midnight and increasing at the midday. This pattern is similar to the variations of traffic volume. The standard deviations in the number of active prefixes for the four time intervals are 76.1, 85.5, 90.5, and 84. Compared with the results of one-hour scale dataset shown in Fig. 4(a), the standard deviations of one-day scale dataset are much larger. The results reveal that the number of active prefixes presents a higher characteristic of local stability. In addition, although the standard deviations in IPv6 network are larger than the observations in IPv4 network [10], the number of active prefixes is still much smaller. Hence, the employment of small-sized routing cache in IPv6 networks is considerable for reducing the hardware cost without compromising the overall performance. 
(a) One Hour

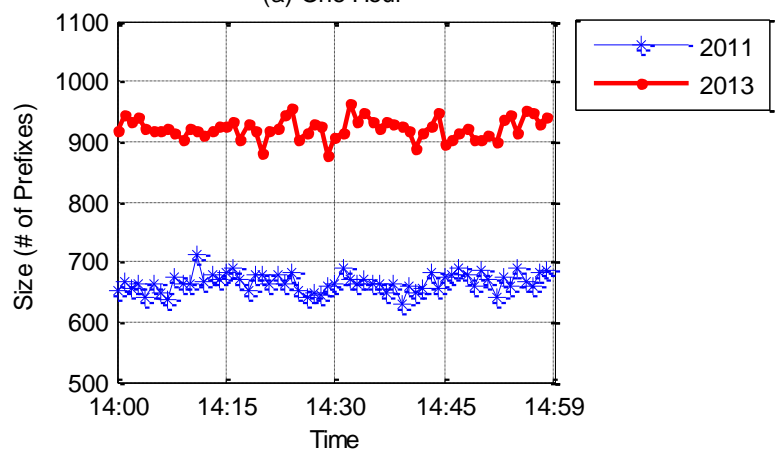

(b) One Day

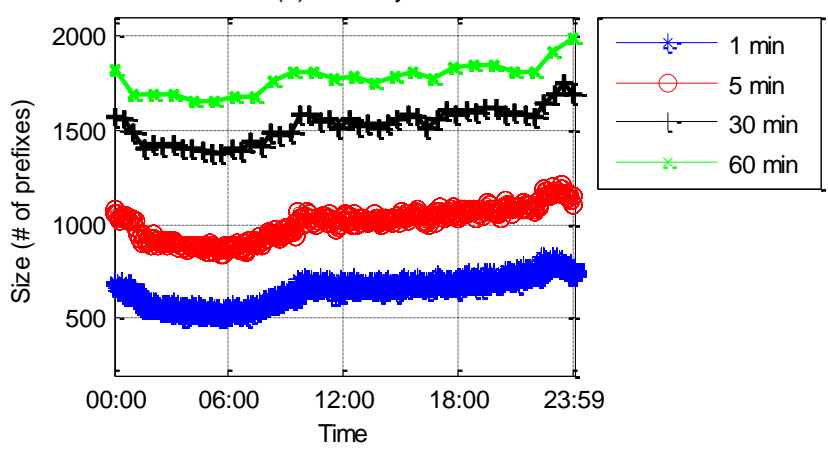

Fig. 4. Active prefixes distribution across different timescales

\subsubsection{Prefixes changes across different timescales}

Changes of the prefixes refer to the prefixes emerging and vanishing between two consecutive periods of time. The number of the changed prefixes is often used to estimate how often the cache should be updated (i.e., cache replacement frequency). In routing cache design, if the replacement frequency is too high, the cache update operation will disturb the per-packet cache lookup operation, compromising the line-rate packet forwarding guarantee. Fig. 5 and Fig. 6 show the number of emerging and vanishing prefixes across different timescales respectively. As depicted in Fig. 5(a) (one-hour scale dataset), the number of emerging prefixes varies from 119 to 174 in 2011, while the number reaches 219 in 2013 according to the one-minute time interval observation. The standard deviations in the number of emerging prefixes are 12 and 19.3 in 2011 and 2013 respectively.

We then observe the same metrics based on the one-day scale dataset (2011/10/24). As shown in Fig. 5(b), results of the larger time intervals (i.e., 30-min and 60-min) change much more than the smaller time intervals (i.e., 1-mim and 5-min). This can be proved by the standard deviations. The standard deviations in the number of emerging prefixes for the four time intervals are 13.3, 15.6, 25.1, and 31.3. In addition, as shown in Fig. 6, the changed trend of vanishing prefixes is similar to the trend of emerging prefixes. The standard deviations observed from the one-hour scale dataset are 11 and 20.6, while from the view of one-day scale dataset, the deviations for the four time intervals are 13.3, 15.3, 23.3 and 34.3. Changes of the prefixes in IPv6 world are small but may be very important because they impact the efficiency of transmission. The emerging prefixes may carry the traffic for important or real-time services, such as financial transactions, video conferences and voice-over-IP, etc. So updating the routing cache instantly is necessary. Our analysis result depicted in Fig. 5 and Fig. 6 has revealed the update frequency. Fortunately, according to our measurements, the prefix update rate is relatively low in this network, which means the prefixes resided in the cache will be quite stable, free from being swapped in and out frequently.

(a) One Hour

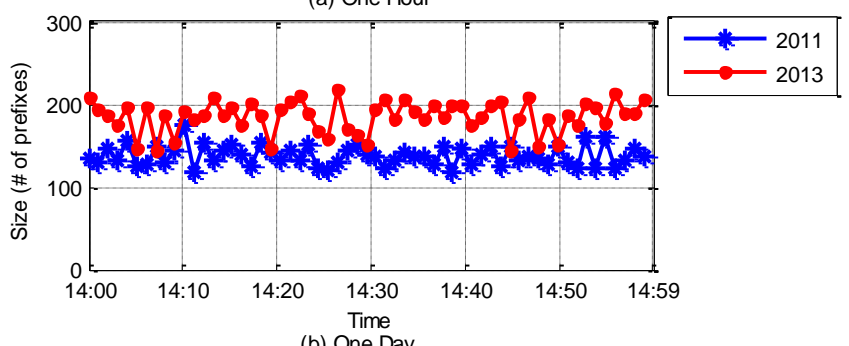

(b) One Day

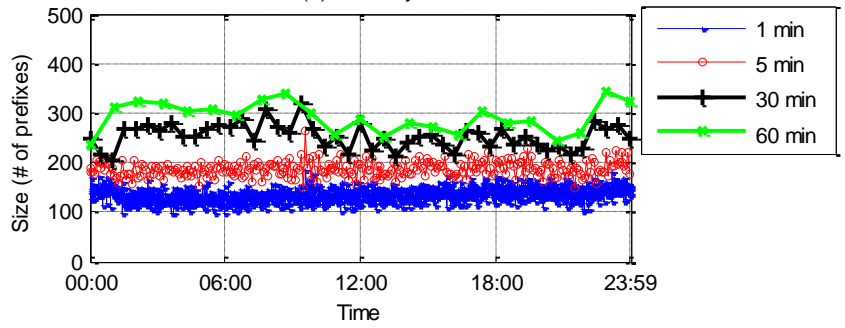

Fig. 5. The number of emerging prefixes across different timescales

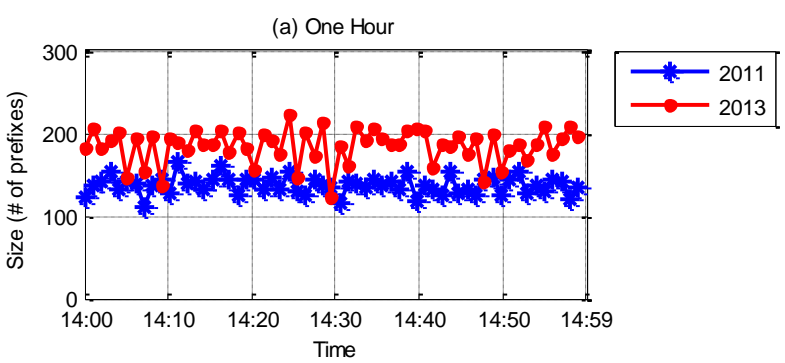

(b) One Day

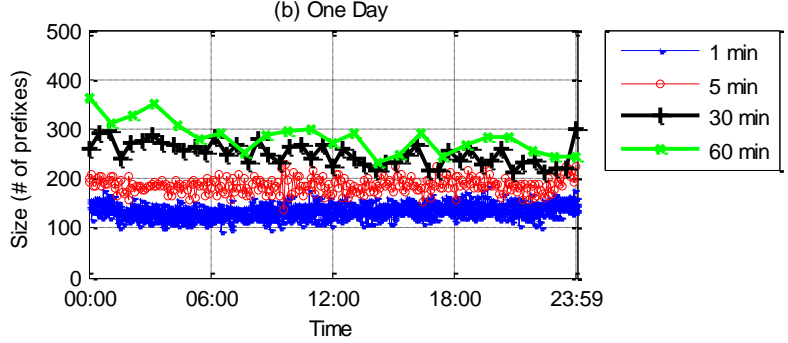

Fig. 6. The number of vanishing prefixes across different timescales 
In addition, we further investigate the bursts of changes, based on which we can gain the information of how large the memory should be reserved for the burst update. Since in modern routers, on-chip buffer is expensive and very limited in size, the burst arrival of requests will potentially cause buffer overflow, leading to the packet miss-forwarding. The burst refers to the maximum value of the emerging prefixes at a time. We define $B(t)$ as the burst of changes observed from time interval $t$. For the one-hour scale dataset, $B_{2011}(1)=174$ and $B_{2013}(1)=219$, while for the one-day scale dataset, $B_{2011}(1)=185, B_{2011}(5)=264, B_{2011}(30)=321$ and $B_{2011}(60)=342$. If the routing cache is updated once an hour and assume that there are no vanishing prefixes, we should reserve 5184 bytes $(342 * 16$ bytes) for the emerging prefixes. If the update frequency of the routing system is higher, the buffer reserved for the worst-case burst updates could be even smaller. There is a trade-off in the on-chip buffer size and the prefix update frequency related to the design of routing protocols (such as BGP and OSPF).

\section{Multihoming Path Diversity and Performance Optimization}

In this section, we first present the multihoming path diversity of IPv6 prefixes, as well as a typical scenario of the studied IPv6 network. Then we show the architecture of MTE, a framework integrating network information monitoring, performance tuning and effect evaluation. Finally, we validate MTE based on the scenario of the studied IPv6 network and show the preliminary validation results.

\subsection{Multihoming path diversity and a typical scenario}

There are a total of 16550 destination prefixes configured on one router of CNGI-6IX, which provides high-speed connection service to domestic CNGI backbones and international research IPv6 networks. As shown in Table 7, prefixes present obvious characteristics of multihoming path diversity. $61 \%$ destination prefixes have more than two paths to reach. Prefixes with two next hops account for $24.1 \%$ of the total prefixes, while the proportion is $31.4 \%$ for prefixes with three next hops.

We use a typical scenario to show the multihoming path diversity. This scenario is about an ISP with alternative external routes for some destination prefixes. As depicted in Fig. 7, CERNET2 has many public peers through the international exchange point of CNGI-6IX. For some destination prefixes, there is more than one route. In the network operation, only one route will be chosen for each destination. However, the chosen routes may not be the best routes. We now explain the reasons for causing this problem. When a new peer interconnects with CERNET2, we will receive a set of destination prefixes that the peer can reach. These destination prefixes provided by the new peer are divided into two parts. 1) For some prefixes, the new peer is the unique next hop to reach them; 2) There are already routes for the other prefixes. CERNET2 is an academic IPv6 backbone, and at present, all the peers it interconnecting with is charging for free, so we care less about commercial profits. By default, the routes with the shortest AS path will be chosen. However, the shortest path may not be the best choice in view of performance. In addition, when a destination prefix has more than one next hop (peer) with the same length of AS path, one of the peers will be selected for the prefix as the next hop randomly. This simplistic processing is not reasonable either.

Due to the multihoming path diversity of IPv6 prefixes and the peering characteristics described in the above scenario, performance optimization is needed for the destination prefixes. As depicted in Fig.7, the bold dashed line represents the ISPs that are chosen as the best next hops for the prefixes with multiple routes, e.g., packets can reach Prefix-2 through many public peers of CERNET2, but currently, ISP-1 is chosen as the best next hop for Prefix-2 because it has the shortest AS path. As an international exchange point, CNGI-6IX can adjust the next hop for each destination prefix to make sure that its member (i.e., CERNET2) can achieve better performance. Note that we do not adjust the next hop for each destination prefix in CERNET2, but in CNGI-6IX. Since most IPv6 networks are operated for academic and experimental goals in the initial phase, performance profits are more concerned about than commercial profits in the current deployment phase of IPv6 networks. Therefore, we conduct performance tuning based on the delay (i.e., RTT) observed from each candidate external route. The commercial decisions of which peers are chosen may involve technical or non-technical factors, which are not addressed in this paper. In addition, performance tuning at prefix granularity is a complicated and error-prone job, especially when many prefixes need to be adjusted. So we put forward a systematic framework of MTE, which makes up the lack of current performance management systems. MTE combine the following four functions together. 1) Benefiting from MTE, many kinds of network information can be gathered and provided for performance tuning. 2) MTE can construct configuration templates and supply the parameters of the templates with definite values. Once necessary, these templates will interact with device and configure the devices automatically, which achieves the goal of automatic performance tuning. 3) MTE can measure and quantify the instability (such as route oscillation, packet delay, packet loss, out-of-order packets, etc.) at the moment of performance tuning, which can instruct us to minimize the influence during the adjustments. 4) MTE also can monitor the performance after the adjustments. So it can continue to optimize the network performance according to the feedback until we can get the satisfying performance profits. Overall, MTE combines the academic research with the real engineering practice and it is a trail towards systematic performance management in IPv6 network. 
Table 7. Multihoming path diversity (2014/5/7)

\begin{tabular}{l|c|c|c|c|c|c|c|c|}
\hline No. of next hop & $\mathbf{1}$ & $\mathbf{2}$ & $\mathbf{3}$ & $\mathbf{4}$ & $\mathbf{5}$ & $\mathbf{6}$ & $\mathbf{7}$ \\
\hline No. of prefixes & 6452 & 3988 & 5200 & 631 & 261 & 15 & 3 \\
\hline
\end{tabular}

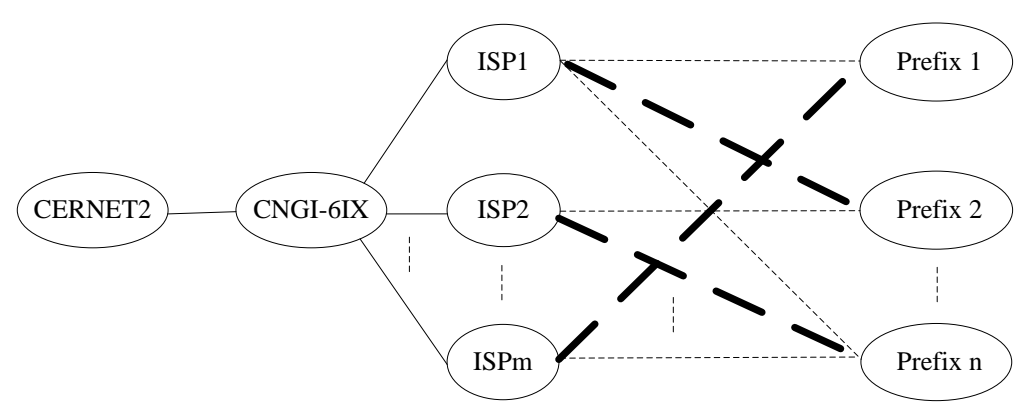

Fig. 7. A typical scenario of the studied IPv6 network

\subsection{Architecture of MTE}

The architecture of MTE is depicted in Fig. 8. It is mainly comprised of three modules, which form a closed loop. In order to show an overview of MTE, we present the basic functionalities of these modules respectively.

\subsubsection{Network Information-Monitoring (NIM) module}

The NIM module is responsible for network information data collection and analysis. The data collection sub-module includes passive measurement and active measurement. Many types of data can be gathered through passive measurement, such as traffic data, routing data, configuration data, etc. Traffic data plays an important role in network information monitoring. There are two main types of traffic data. One is flow-level data collected by enabling Netflow/IPFIX feature in routers. The other is packet-level data captured through port mirroring or optical splitter. Currently, due to the huge amount of traffic data, Netflow is widely used in traffic analysis. Routing data includes statistical routing data (i.e., routing information base tables and forwarding information base tables) and dynamic routing data (i.e., update messages and withdraw message). We gather the statistical routing data through automated scripts, which encapsulate console commands and expect [40] syntax. We gather the dynamic routing data from a BGP daemon, developed based on Zebra [41]. Configuration data mainly refers to the configuration files gathered from the switches and routers. We deploy rancid [42], which can download and upload configuration files from network devices periodically. In addition, the tool of $S V N$ [43] is used to check the differences between different versions of configuration files. There are many tools for active measurement, such as ping, traceroute, mtp, etc. We also develop some active measurement tools based on different protocols, such as UDP, TCP, HTTP, etc. Many methods can be used to collect network information data, so we can choose the optional methods according to the requirements. The data analysis sub-module analyzes the data collected by passive and active measurement. Many metrics can be investigated, such as routing updates, delay, packet loss, etc. All these analysis results can be provided for the upper modules.

\subsubsection{Network Performance-Tuning (NPT) module}

The NPT module consists of two sub-modules: template creation and template provisioning. The template refers to the configuration templates, each of which contains a set of configuration commands and represents a specific functionality or an explicit configuration task. Operators are faced with many problems involving in performance management during their daily operations. The typical problems are routing related, for example, adjusting the parameters of OSPF and BGP for load balancing and performance improvement. The template creation sub-module can construct configuration templates through correlating high-level configuration tasks with device-level configuration commands. The templates can be reused when necessary. So they can greatly lighten the burden on operators. According to the data analyzing results provided by NIM module, the template provisioning first check whether performance tuning is needed or not. Once the performance tuning is determined to conducted, this sub-module will transform the configuration templates into executable scripts, which can configure the task on the corresponding devices automatically. In addition to the primary functionality, the template provisioning sub-module also provides a web interface for configuration information interaction. The interface is multifunctional, i.e., operators can not only perform configuration operations on devices, but also show and check configuration information through this interface. 


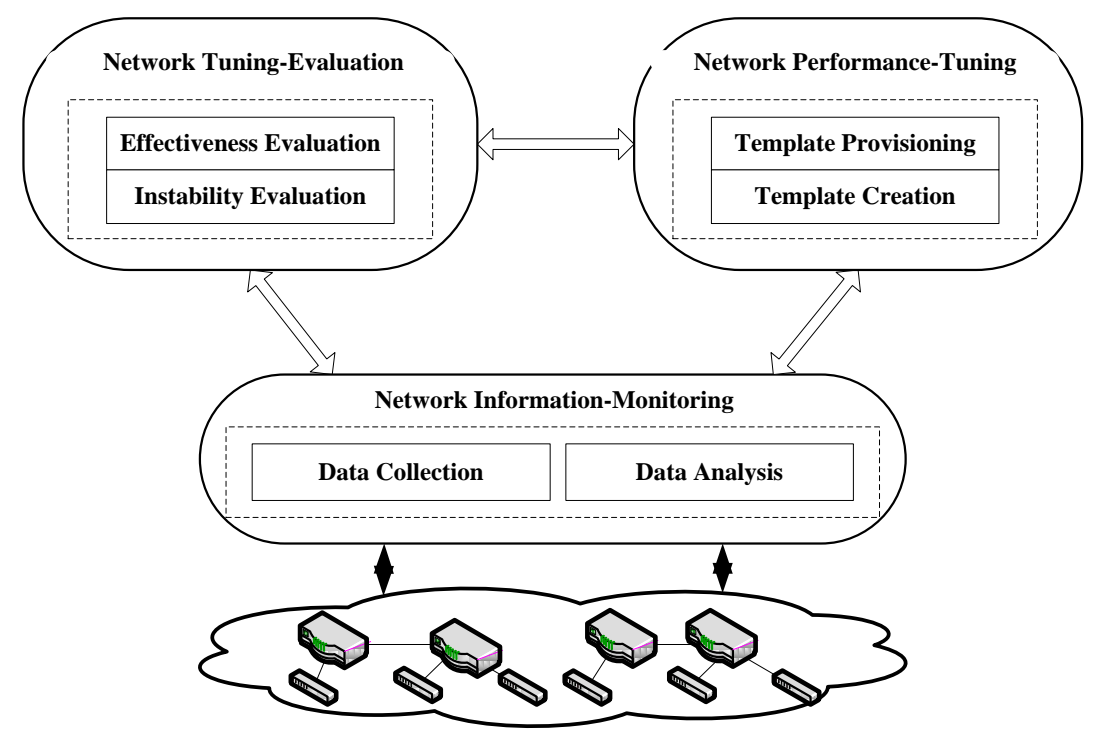

\subsubsection{Network Tuning-Evaluation (NTE) module}

Fig. 8. Architecture of MTE

The NTE module has the ability of evaluating the effect during and after performance tuning. When we adjust values of parameters, such as cost of OSPF, local preference of BGP, etc., network performance will be influenced instantly. For example, when we set a backup next hop with the highest local preference for a prefix, some packets of the prefix may lose or be in disorder during the routing convergence. The instability evaluation sub-module is used to analyze the influence caused by network adjustments. We quantify the influence and further try to decrease the influence. For example, we may find the optimal time for adjustments. We may also find the optimal adjusting order when there are many prefixes that are needed to be adjusted. The effectiveness evaluation sub-module is used to analyze the results after the performance tuning. Performance will not be improved even if we have a detailed analysis and plan before the optimization. Therefore, we need a timely feedback after the performance tuning. If we cannot get the expected results for some prefixes, i.e., the performance after the tuning is not better than that before the tuning, we will adjust these prefixes repeatedly until we get the expected results. Of course, we also may roll back configurations for some prefixes to the state before the first tuning.

\subsection{Validation on MTE}

Benefiting from the functions and methodologies defined in each module of MTE, we can optimize the performance of the scenario described in the studied IPv6 network by the following four procedures: testing, calculating, adjusting and evaluating. Procedures of testing and calculating are mainly processed by NIM module. Procedures of adjusting and evaluating are mainly processed by NPT module and NTE module respectively. The layout of our validation on MTE is shown in Fig. 9.

(a) CERNET2 - AS 63910
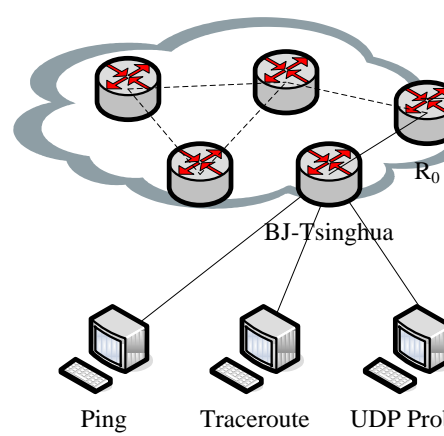

(b) CNGI-6IX - AS 63911 (c) AS 43948

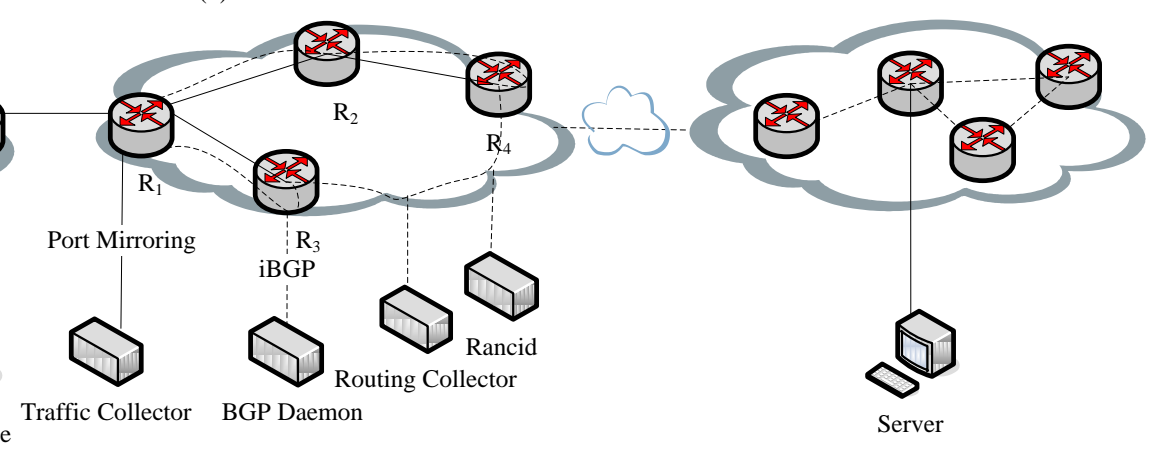

Fig. 9. Layout of our validation on MTE

\subsubsection{Testing}


We first measure the performance of the destination prefixes with alternative paths. We adopt the route injection technique [44] to measure the performance for each prefix, which can significantly reduce the influence on current traffic transmission. For example, to measure an alternative path for a prefix, we select an active $i p$ within the prefix and configure a route for this single $i p$ $(i p / 128)$ in the egress router. Since BGP follows the principle of longest prefix match, only the packets destined to $i p$ will go through the configured route while the rest of the traffic will continue to use the current route.

The NIM module provides support for the testing process from four aspects. Firstly, as depicted in Fig. 9(b), the routing collector can gather the statistical routing data, i.e., RIBs and FIBs. We then pick out the destination prefixes with multiple paths from the RIBs. Secondly, the traffic collector shown in Fig. 9(b) can gather the packet-level traffic data, according to which, we can collect active addresses for the destination prefixes. Thirdly, the BGP daemon maintains iBGP sessions with all the routers of CNGI-6IX (AS 23911, IPv6 International eXchange). The iBGP sessions are a full mesh map. In Fig. 9(b), we use dotted lines to simply represent the connections of iBGP sessions. The BGP daemon is responsible for the route injection. Fourthly, as shown in Fig. 9(a), ping is used to measure the RTT of each destination address and traceroute is used to check whether the path is changed for an address after the route injection. The testing process can be divided into the following five steps.

Step-1: List the best and backup eBGP routes for each destination prefix according to the RIB of one border router. As depicted in Fig. 9(b), the RIB of $R_{3}$ is utilized in our study. The prefixes that have only one eBGP route will be removed. The remainders will be sorted and stored in the set of Pre.

Step-2: For each prefix from the set of Pre, for example Pre $e_{1}$, we record the length of Pre $_{1}$, marked with $L$. We collect the active IP addresses within $\mathrm{Pre}_{1}$ and then randomly select $X$ addresses in the same subnet (i.e., with the same first 64 bits of the address) for each alternative path (including the current best path). Note that $X$ is the number of next hops of Pre $_{1}$.

Step-3: The BGP daemon sends crafted routes (host routes) on demand to all the routers maintaining iBGP sessions with it. After the route injection operations, packets going to the selected addresses will be forwarded along each alternative path of Pre $_{1}$. We conduct ping and traceroute to each destination address. The results of the traceroute are used to verify that packets are correctly transmitted through the expected paths. The average RTT of each address is calculated based on the ping results. Then the average RTT of each path is recorded in the set of $T_{p r e}=\left\{T_{1}, T_{2}, \ldots, T_{m}\right\}$, where $T_{m}$ represents the average RTT from the test client to $\mathrm{Pre}_{1}$ through path M.

Step-4: The BGP daemon withdraws the crafted routes from all the routers maintaining iBGP sessions with it.

Step-5: Repeat step-2 to step-4 until all the prefixes in the set of Pre are measured. At last, we get the set of $\mathrm{T}_{\mathrm{pre}}=\left\{\mathrm{T}_{1}, \mathrm{~T}_{2}, \ldots, \mathrm{T}_{\mathrm{m}}\right\}$ for each prefix with multiple next hops.

\subsubsection{Calculating}

We calculate the total performance profits that can be achieved based on testing results. This work is done by the NIM module. Here, we define two variables that will be used in the whole evaluation process. $T_{x}$ is the delay from the test client to the prefix through the default (current best) route. $\mathrm{T}_{\mathrm{y}}$ is the minimum delay from the test client to the prefix through the backup eBGP routes.

$$
\text { If } \mathrm{T}_{\mathrm{x}}<\mathrm{T}_{\mathrm{y}} \text {, then } \mathrm{E}_{1}+=(64-\mathrm{L})\left(\mathrm{T}_{\mathrm{y}}-\mathrm{T}_{\mathrm{x}}\right)
$$

If the delay of the default route is smaller than the minimum delay of the backup eBGP routes, it means that routing policy for this prefix does not need to be adjusted. We denote L the length of the prefix. As depicted in equation (I), we accumulate the differences of the whole address space of this prefix to $E_{1}$. The larger the value of $E_{1}$ is, the fewer requirements of routing adjustments need to be processed.

$$
\text { If } T_{x}>T_{y} \text {, then } E_{2}+=(64-L)\left(T_{x}-T_{y}\right)
$$

If the delay of the default route is larger than the minimum delay of the backup EBGP routes, it means that routing policy for this prefix needs to be adjusted. As depicted in equation (II), we accumulate the differences of the whole address space of this prefix to $E_{2}$. The larger the value of $E_{2}$ is, the more requirements of routing adjustments need to be processed.

$$
\mathrm{E}=\mathrm{E}_{2}-\mathrm{E}_{1}
$$

As shown in equation (III), we define $\mathrm{E}$ as the difference between $\mathrm{E}_{2}$ and $\mathrm{E}_{1}$. The value of $\mathrm{E}$ represents the total performance profits that can be gained through adjusting routing policies. Operators will determine whether they should change routing policies based on the value of $\mathrm{E}$. If $\mathrm{E}<=0$, it means that there is no need for performance improvement. While, providing $\mathrm{E}>0$, the larger the value of $\mathrm{E}$ is, the more requirements of routing policies need to be adjusted. Routing adjustments across the whole network will cause great influence on the network, as well as may bring in misconfigurations. So we will adjust the routing policies only after we can affirm that we can gain obvious performance profits.

\subsubsection{Adjusting}


The adjusting process is done by NPT module. According to the results analyzed by NIM module, if we can gain obvious performance profits, we will conduct performance tuning. For each prefix in the set of Pre, if $\mathrm{T}_{\mathrm{x}}>\mathrm{T}_{\mathrm{y}}$, we will adjust the route with the minimum delay (among all the backup routes) as the new default route. This adjustment is realized by setting the localpreference of the route with the highest value among all the routes. Note that all the adjustments are conducted under the restriction that the aggregate traffic of each path should meet the link capacity. Template creation sub-module of NPT module is responsible for creating configuration template based on the configuration information gathered by Rancid (As shown in Fig. 9(b)). Then the template provisioning sub-module encapsulates the configuration template into scrip with expect [40] syntax, which can interact with router and configure commands on router automatically. There are three types of operations on routing policies (i.e., adding, modifying and deleting a routing policy) to change the next hop for a prefix or a set of prefixes. Given a prefix of $2 \mathrm{~A} 02: 750:: / 32$ that needs to be adjusted, we illustrate the processes of template creation and template provisioning, as well as instantiate the parameters of the templates with definite values under the environment of Cisco IOS Release 12.0(32)SY16.

Step-1: According to the RIB information, we identify and record the AS number, neighbor address, and local-preference for both the current next hop and the new next hop. We use $N_{i}$ (AS number, neighbor address, local-preference) to represent the $\mathrm{i}_{\text {th }}$ next hop of a prefix. RIB information is gathered by the routing collector. As shown in Fig. 10(a), we want to change the next hop for prefix of $2 \mathrm{~A} 02: 750:: / 32$ from $N_{1}(6939,2001: 252:: 105,90)$ to $N_{2}(6939,2001: 7 \mathrm{FA}: 0: 1:: \mathrm{CA} 28: \mathrm{A} 19 \mathrm{E}, 80)$. Note that $N_{l}$ and $N_{2}$ belong to two different border routers of AS 6939.

Step-2: With the access permission of $R_{3}$ shown in Fig. 9(b), we check the name of the import routing policy of $N_{2}$ through the web interface provided by NPT module. The query command is show bgp neighbors 2001:7FA:0:1::CA28:A19E | include Route map. The name of the import routing policy of $N_{2}$ is ebgp-v6-he-im-policy.

Step-3: According to the name of ebgp-v6-he-im-policy, we extract corresponding configurations of the import routing policy of $\mathrm{N}_{2}$ from the configuration information gathered by Rancid. Configurations of ebgp-v6-he-im-policy are shown in Fig. 10(b).

Step-4: Based on the above information, we create a configuration template to change route to the new next hop. The configuration template is shown in Fig. 10(c). We should consider the following aspects when we create the template and determine the values of the parameters.

Firstly, the sequence number of the route-map should be selected carefully. As shown in Fig. 10(b), we can choose the value from 21 to 89 at first sight. However, in order to guarantee the new route-map taking effect, we must check the prefix-list of self-v6 (line 1 - line 3) and the as-path-access-list of as-path 90 (line 4 - line 6). The query commands are show ipv6 prefix-list self-v6 and show ip as-path-access-list 90 respectively. Query results are used to check whether deny 10 and deny 20 are related to prefix of 2A02:750::/32 and AS path of the new path (i.e., 6939 43948). If prefix-list of self-v6 includes $2 \mathrm{~A} 02: 750:: / 32$, the value of the new route-map must be selected from 1 to 9 . If prefix-list of self-v6 does not include 2A02:750::/32, but as-path-access-list of as-path 90 includes 6939 or 43948 , the value of the new route-map must be selected from 11 to 19 . We have checked that adjusting the next hop of $2 \mathrm{~A} 02: 750:: / 32$ to $N_{2}$ is not restricted by deny 10 and deny 20, so we confirm that we can select the value for the new route-map from 21 to 89 . We select 80 for the new route-map as shown in line 2 of Fig. 10(c).

Secondly, the value of local-preference should be set correctly. As shown in Fig. 10(a), the local-preference of the current best route is 90 , so we should choose a value larger than 90 for the local-preference of the new route. We choose 100 for the new route as shown in line 4 of Fig. 10(c).

Thirdly, the configuration template depicted in Fig. 10(c) is commonly used. If we want to apply this template to other prefixes that needed to be adjusted to the same or different next hops, we just need to determine and replace the values of the variables underlined in Fig. 10(c).

Step-5: In order to reduce the workload and the possibility of misconfigurations, we transform the configuration template into a script (shown in Fig. 11(a)) that can connect to the corresponding router and execute the configuration commands automatically. The automated execution benefits from the functionality of expect. Note that all the characters underlined can be regarded as variables, the values of which can be got from a file through a shell program.

Our previous work has described the thought of template extraction from configuration files [48]. In this paper, we put the idea into practice, which combines requirements of performance tuning with experience of operators. 


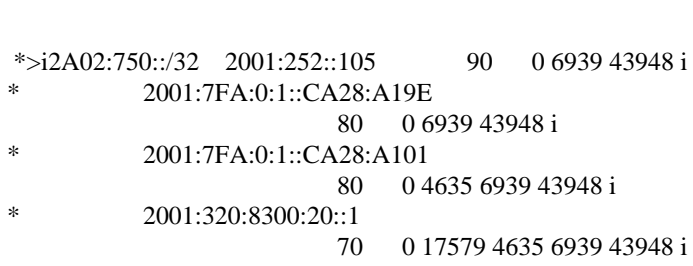

\subsubsection{Evaluating} NIM module. this. Fig. 11(c). (a) Example of template provisioning

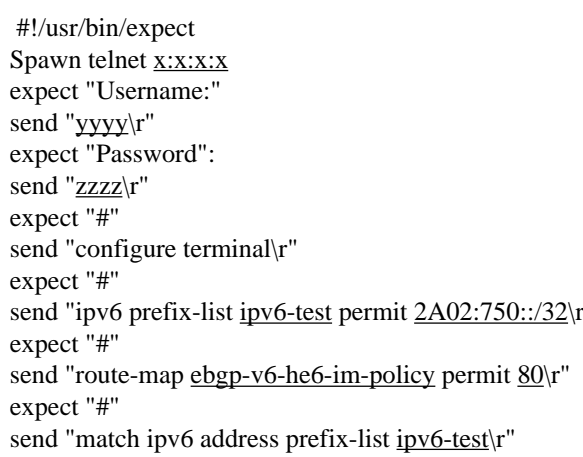

\subsection{Preliminary validation results}

1 route-map ebgp-v6-he6-im-policy deny 10 2 match ipv6 address prefix-list self-v6 3 !

4 route-map ebgp-v6-he6-im-policy deny 20 5 match as-path 90

6 !

7 route-map ebgp-v6-he6-im-policy permit 90

8 match ipv6 address prefix-list valid-v6

9 set local-preference 80

10 set community 23911:6939 additive
1 ipv6 prefix-list ipv6-test permit 2A02:750::/32

2 Route-map ebgp-v6-he6-im-policy permit $\underline{80}$

3 match ipv6 address prefix-list ipv6-test

4 set local-preference $\underline{100}$

5 set community 23911: $\underline{6939}$ additive

6 exit

7 exit

8 clear bgp 2001:7FA:0:1::CA28:A19E soft in

9 wri

Fig. 10. Example of template creation

The evaluating process is used to evaluate the instability during the adjustments and the effectiveness after the adjustments. Evaluating is mainly scheduled by NTE module, as well as supported by NIM module.

On the one hand, in order to gain more performance profits, we adjust the local-preference of some destination prefixes from one route to another. Although BGP can adapt to these adjustments, it takes time to propagate the necessary update messages throughout the network. In summary, configuration changes of routing policies will introduce BGP route updates. In addition, during this convergence period, some packets related to the adjusted prefixes are already on the road to their destinations and new packets are constantly sent out. These packets may encounter delay, loss, etc. As depicted in Fig. 9(a), we deploy some active measurement tools (i.e., ping, traceroute and UDP probe) to measure the performance during the routing adjustments. We also observe routing updates (BGP daemon) and configuration changes (Rancid) through passive measurement. As shown in Fig. 8, the sub-module of instability evaluation in NTE module launches the measurement tools and aggregates the data analysis result in

On the other hand, after the first performance tuning, we will further evaluate the performance of the new paths for the adjusted prefixes. The effectiveness evaluating process is similar to step-3 of the testing process. For an adjusted prefix, if the delay of the new path is not better than the old path, we will continue to adjust this prefix to other alternative paths, or reuse the original path as the best route. As depicted in Fig. 8, the sub-module of effectiveness evaluation in NTE module is responsible for

In the evaluating process, we not only need to add routing policies, but also need to modify or delete routing policies to control the selected path. Fig. 10(c) is a template of adding a routing policy. If we intend to adjust the prefix to the original path, we just need to decrease the value of the local-preference of the added routing policy. Corresponding template of modifying a routing policy is shown in Fig. 11(b). In addition, when we intend to disable a routing policy, we can use the template as shown in

expect "\#"

send "set local-preference $100 \backslash \mathrm{r}$ "

expect "\#"

send "set community 23911: 6939 additivelr"

expect "\#"

send "exit\r"

expect "\#"

send "exitłr" expect "\#"

send "clear bgp 2001:7FA:0:1::CA28:A19E soft in\r" expect "\#"

send "wrilr"

expect "\#" (b) Template of modifying a routing policy

1 route-map ebgp-v6-he6-im-policy permit $\underline{80}$

2 match ipv6 address prefix-list ipv6-test

3 set local-preference $\underline{80}$

4 set community 23911: $\underline{6939}$ additive

5 exit

6 exit

7 clear bgp 2001:7FA:0:1::CA28:A19E soft in

8 wri

(c) Template of deleting a routing policy

1 no route-map ebgp-v6-he6-im-policy permit $\underline{80}$

2 exit

3 clear bgp 2001:7FA:0:1::CA28:A19E soft in

4 wri

Fig. 11. (a) Example of template provisioning; (b) Template of modifying a routing policy; (c) Template of deleting a routing policy 
A network-wide testing and adjustment will greatly influence the network. Hence, we select 9 prefixes with alternative routes to conduct a preliminary validation on MTE. Among the 9 destination prefixes, three prefixes have 4 alternative paths, while the others have only 2 alternative paths. The 9 prefixes belong to IPv6 enabling hosting providers [45].

The testing results for the selected 9 prefixes are shown in Table $8 . T_{1}$ is the average RTT of current best route, while $T_{2}$ is the minimum average RTT of the alternative paths. According to the testing results, we calculate the performance profits gained from the 9 prefixes, $\mathrm{E}_{1}=12160, \mathrm{E}_{2}=704, \mathrm{E}=11456$. The results reveal that it is worth conducting optimization for some of these prefixes. We adjust 8 prefixes to other alternative routes with better performance. We also evaluate the 8 adjusted prefixes after the tuning. The results are similar to the results observed in the testing process. Through the effectiveness evaluation, we could determine that our adjustments are reasonable and beneficial.

Table 8. Testing results of the 9 prefixes

\begin{tabular}{c|c|c|c|c|c|c|c|c|c|c}
\hline Prefixes & $\mathbf{1}$ & $\mathbf{2}$ & $\mathbf{3}$ & $\mathbf{4}$ & $\mathbf{5}$ & $\mathbf{6}$ & $\mathbf{7}$ & $\mathbf{8}$ & $\mathbf{9}$ \\
\hline $\mathbf{T}_{\mathbf{1}}(\mathbf{m s})$ & 374 & 267 & 265 & 407 & 386 & 375 & 376 & 391 & 297 \\
\hline $\mathbf{T}_{\mathbf{2}}(\mathbf{m s})$ & 318 & 239 & 235 & 341 & 328 & 346 & 319 & 335 & 319 \\
\hline
\end{tabular}

Routing adjustments will greatly influence the network operation. Therefore, we need an evaluation on the instability caused by performance tuning. We first understand the influence, and further try to find out how to decrease the influence. We use many metrics to understand the instability, such as routing updates, packet delay, loss, and out of order packets. Then we have the basis and confidence to conduct network-wide adjustment for performance improvement. As depicted in Fig. 9(c), we choose a prefix (2A02:750::/32) in AS 43948, which can provide IPv6 VPS (Virtual Private Server). We rent a server from AS 43948 and deploy a program in the remote server. The server can receive UDP packets sent from the hosts in CERNET2 and record the process of packet reception. We also use ping to measure the performance, traceroute to check path changes, and BGP daemon to monitor routing updates. As depicted in Fig. 12, there are four routes from CNGI-6IX to AS 43948. Note that $p_{1}$ and $p_{4}$ are two different routes going to AS 43948. Currently, the best route is represented by $p_{1}$ in Fig. 12. Applying the testing methodologies described in Section 4.3.1, we measure the average RTT of the four routes. According to the testing results shown in Table 9, we decide to adjust $p_{4}$ as the new best route for prefix of $2 \mathrm{~A} 02: 750:: / 32$. We now measure and unveil the instability during the adjustment.

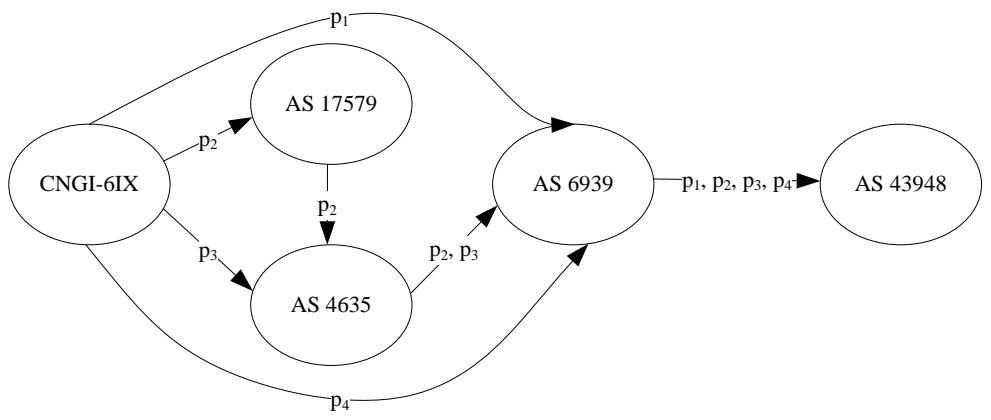

Fig. 12. Routes from CNGI-6IX to AS 43948

Table 9. Average RTT of the four routes

\begin{tabular}{c|c|c|c|c}
\hline Path & $\mathbf{p}_{\mathbf{1}}$ & $\mathbf{p}_{\mathbf{2}}$ & $\mathbf{p}_{\mathbf{3}}$ & \multicolumn{1}{c}{} \\
\hline $\mathbf{T}_{\mathbf{1}}(\mathbf{m s})$ & 408.1 & 350.4 & 351.8 & 350.3 \\
\hline
\end{tabular}

\subsubsection{Routing updates}

As depicted in Fig. 9(b), eBGP peer relationship is created between $R_{3}$ and BGP daemon, so the BGP daemon can receive all the update and withdraw messages from $R_{3}$. Through our analysis, only one update message is triggered and sent by $R_{3}$ when we adjust the path from $p_{1}$ to $p_{4}$. The reason is that when we change the next hop for a prefix from $A$ to $B$, the computational complexity is $O(1)$ [46]. In our experiment, $A$ and $B$ are two different border routers of AS 6939. They connect with different routers of CNGI-6IX. The results illustrate that only the incremental routing dynamics are triggered and propagated, and the amount of the update messages depends on the number of prefixes that will be adjusted.

\subsubsection{Packet delay and loss}

Although we want to improve the performance of the packet transmission, average RTT of the packets during the optimization may be a little higher than that under the normal transmission. In addition, packets on the road may encounter packet loss at the time of adjusting. RTT is measured by ping (/100ms) and pack loss is observed by UDP probe (/50ms). Results show that no packets encounter packet loss, and only one packet is obviously delayed during the adjustment. The RTT of that packet is $488 \mathrm{~ms}$, 
which is higher than the values before $(408.1 \mathrm{~ms})$ and after $(360.3 \mathrm{~ms})$ the adjustment. The results reveal that the influence on packet delay and loss is not obvious. Two reasons may explain the results observed. For one thing, the topology of CNGI-6IX is simple. Routers can quickly converge in a stable state after the adjustment. For another thing, whenever there is a change in the routing policy, the BGP session must be reset (cleared) for the new policy to take effect and the routing table to be reconfigured. As depicted in the line 8 of Fig. 10(c), we use the BGP soft reset, which allows routing policies to be reconfigured and activated without clearing the BGP session.

\subsubsection{Out of order packets}

We send UDP packets with sequence number to the server. The server will accept the probes and distinguish the order of the received packets. Therefore, we can analyze out of order packets during the adjustment based on the receptions of UDP probes. We find that three packets are disordered during the adjustment. We also calculate the reordering offset of the out of order packets We denote reordering offset the difference between the actual arrival order and the expected arrival order of an out of order packet [35]. For example, for received packets with sequence number of $\{1,3,4,5,2,6\}$, packet 2 is out of order, because 2 is smaller than the expected sequence number of 6 . The reordering offset is $6-2=4$. The maximum reordering offset of the disordered packets in our experiment is 4 . The reason for the reordering packets is that some packets on the original path are delayed during the adjustment, while the subsequent packets on the new path reach the server first.

According to our observation, we conclude that routing can converge quickly during our adjustment. The situation of packet delay, loss and disorder is not serious. However, our evaluation is based on adjusting one prefix and the influence is observed from some testing clients and a remote server. Once we adjust a set of prefixes and some prefixes are carrying heavy background traffic, the influence will be enlarged. In addition, other adjustments, such as withdrawing a route of a prefix to an ISP [35], will influence the network more obviously. Therefore, we should take notice of the influence when we conduct large scale adjustments for performance improvement. Firstly, we should notice that routing updates show a liner relation with the number of prefixes to be adjusted. So in order to escape from serious routing oscillation, we should adjust a small set of prefixes once a time. Secondly, although packet loss is not found, packet delay and disorder are discovered during the routing adjustments. When the background traffic of the adjusted prefixes is heavy, or part of the background traffic is related to real-time services (such as VoIP, financial transaction, etc.), we should adjust these prefixes in an idle time.

\section{Discussion}

We now discuss two key implications of our observations on prefix-related characteristics of the studied IPv6 network, as well as the validation results of MTE. One is network management and routing cache design in IPv6 world. The other is multi-source measurements and advance planning for performance tuning.

\subsection{Network management and routing cache design}

As depicted in Fig.2, a small proportion of prefixes account for most of the traffic and packets. Therefore, when supervision of the IPv6 network becomes necessary, these prefixes may be the priority ones to be monitored by operators. Currently, network management changes from coarse-grained to fine-grained. Hence, understanding prefixes-related characteristics and stepping towards prefixes-granularity management are essential in IPv6 networks. For example, some popular prefixes may carry traffic for some important real-time services. We can not only set higher priority to these prefixes to get the routes with the best performance, but also cache these prefixes in TCAM, then packets to these destination prefixes can be forwarded efficiently. In addition, we can configure redundant links for links (carrying traffic of these prefixes) which may be congested or broken down in advance, when failures happen, the network can reroute the traffic of these prefixes to other links or devices automatically. However, this requires us to have a more comprehensive understanding of the traffic characteristics of the important prefixes. We leave further analysis of the main prefixes as our future work.

As in shown in Table $2 \sim$ Table 4, the length of active prefixes mainly distributes in the interval of [32, 48] in IPv6 world. This is a very important property since most fast-speed forwarding hardware is of fixed word width to store the table index, and we can slice the TCAM address space into 48-bit index for per prefix to hold the most frequently accessed IPv6 records, leaving other less popular records on secondary storage. This scheme can also be combined with software-based prefix lookup scheme via searching on trie structure, with most traffic processing offloaded to exact matching via hashing, which will be much faster than trie traversing with multiple memory accesses. As shown in Fig. 2 and Fig. 3, we can conclude that most traffic and packets are contributed by a small part of prefixes and addresses. There are two implications from this observation. For one thing, since traffic and packets distribution is highly skewed, routing cache can be a straightforward yet practical optimization in IPv6 world. For another, due to a small part of IPv6 addresses account for most packets, we can add host routing items in routing cache, and then packets to these destination addresses can be forwarded efficiently. As depicted in Fig. 4, active prefixes are small and stable across different timescales. Therefore, the size of the routing cache is considerably small, which reduces the system budget when using TCAM as the cache storage. 


\subsection{Multi-source measurements and advance planning}

In Section 4, we focus on validating MTE based on a typical scenario in the studied IPv6 network. The validation is strongly supported by NIM module, which provides multi-source measurements. Based on the open-source tools and self-developed systems (such as traffic collector, routing collector, Zebra, Rancid, Ping, Traceroute, UDP Probe, etc.), we can get network information from many sources. For example, we can use traffic collector to collect IP packets and further extract active addresses for our validation on MTE. We also can analyze traffic characteristics and user behaviors based on the IP packets to balance traffic transmission and improve user experience. The typical scenario adopted in this paper is just a case use of MTE. That is to say, MTE can optimize network performance from many aspects on the basis of multi-source measurements. In addition, Multi-source measurement provides real-time monitoring. On one hand, it can monitor the network performance after any adjustment. The information can be fed back to validate the effectiveness of adjustments, as well as instruct us to continue to adjust routing polices until we can get the best performance. On the other hand, it can monitor the network dynamics (such as routing updates, traffic patterns, packet delay, loss and disorder, etc) caused by routing adjustments. The dynamical information is crucial for network adjustments, because it can instruct how to decrease or avoid the influence caused by adjustments for performance improvement.

MTE can not only monitor and optimize the network performance, but also evaluate the insanity caused by routing adjustments. As stated in Section 4.4, routing adjustment in our experiment has brought in routing updates, packet delay increase and out of order packets. The influence will be more obvious when the scale of the adjustments is large. Therefore, we must make plan for routing adjustments in advance. For example, based on the data gathered by traffic collector, we can monitor traffic variations for all the prefixes. Traffic often presents diurnal pattern, so we can get the timescales with the least traffic for each prefix. We divide the prefixes into different groups according to the timescales. Prefixes in the same group will be adjusted simultaneously. However, the time-scale of the division and the upper limit of the prefixes in a group still need to be confirmed. In addition, we should further identify the prefixes carrying real-time services and provide class of service at prefix granularity. For example, if a prefix belongs to a trading platform, we should adjust the prefix to another path when no user traffic distributes on that prefix. However, there may be no optimal adjustment timescales for this prefix, so we should contact the operators of the trading platform and appoint a time for adjustment.

\section{Conclusions and Future Remarks}

IPv6 is experiencing fast development as the next generation network. But our understanding of IPv6 cannot keep up with the growth of it. In this paper, we first try to understand the characteristics of IPv6 at prefix-granularity mainly based on RIR data and the traces of CERNET2. There are three main findings from our observations. 1) Most active prefixes are in the length from 32 bits to 48 bits. Hence, 48-bit width TCAM entry will be sufficient to cache the prefixes that account for a majority of the traffic. 2) Traffic distribution across prefixes is highly skewed in the studied network. This characteristic is strongly supportive for introducing routing cache in IPv6 world. 3) The size of prefixes across different timescales and the number of changing (emerging and vanishing) prefixes are relatively stable. Therefore, prefixes in the cache need not to be swapped in and out frequently. Deep analysis shows that if the update frequency is one hour, we only need to reserve 5184 bytes memory for the worst-case update. Overall, our analysis results not only reveal the characteristics of an IPv6 network at prefix-granularity, but also provide basis for routing cache design in IPv6 world.

In addition, due to the multihoming path diversity of destination prefixes and the real network management requirements of the studied IPv6 network, we propose and validate a framework of MTE, which integrate information monitoring, performance tuning and effect evaluation. Firstly, MTE can collect and analyze many kinds of data from both passive and active measurement. These multi-source measurements provide fundamental basis for performance tuning and effect evaluation. Secondly, from the practice standpoint, it can conduct performance tuning in an automated way. Thirdly, MTE can not only optimize the network performance according to the feedback repeatedly, but also evaluate the instability caused by network adjustments. Note that MTE is a systematic framework for performance management, not only applying to IPv6 networks, but also applying to IPv4 networks.

We will collect more traces from different IPv6 networks and conduct comparison and generalization analysis in the future. We will also validate MTE on more scenarios in the actual network management and evaluate the instability caused by performance tuning under other situations of the studied IPv6 network, such as modifying a policy and deleting a policy to control the outgoing packets, withdrawing a route of a prefix to an ISP to control the incoming packets, etc.

\section{ACKNOWLEDGMENTS}

The authors would like to thank Zhiyan Zheng for his aid in gathering data and revising this paper. We also thank anonymous reviewers for their constructive comments. This work is supported by the National Science Foundation for Distinguished Young Scholars of China under Grant No. 61225012 and No. 71325002; the National Natural Science Foundation of China under Grant 
No. 61572123; the Specialized Research Fund of the Doctoral Program of Higher Education for the Priority Development Areas under Grant No. 20120042130003.

\section{REFERENCES}

[1] Internet Society, Internet Society Statement on IPv4 Depletion, http://isoc.org/wp/newsletter/?p=3139.

[2] APNIC, Key Turning Point in Asia Pacific IPv4 Exhaustion, http://www.APNIC.net/data/assets/pdf_file/0018/33246/Key-Turning-Point-in-Asia-PacificIPv4-Exhaustion_English.pdf.

[3] S. Deering and R. Hinden, "Internet Protocol, Version 6 (IPv6) Specification", RFC 2460, 1998.

[4] M. J. Akhbarizadeh, M. Nourani, R. Panigrahy, and S. Sharma. A TCAM-based parallel architecture for high-speed packet forwarding. Computers, IEEE Transactions on, 2007, 56(1), pp.58-72.

[5] BGP Routing Table Analysis Report. http://bgp.potaroo.net/.

[6] J. Rexford, J. Wang, Z. Xiao and Y. Zhang. BGP routing stability of popular destinations. In Proceedings of the 2nd ACM SIGCOMM Workshop on Internet measurment. 2002, pp. 197-202.

[7] "How to Choose the Best Router Switching Path for Your Network," August 2005. Cisco Systems, http://www.cisco.com/image/gif/paws/13706/20.pdf.

[8] C. Partridge, P. Carvey, et al., “A 50-Gb/s IP router,” in IEEE/ACMTrans. Networking, 1998.

[9] L. Iannone and O. Bonaventure, "On the Cost of Caching Locator/ID Mappings," in Proc. ACM CoNEXT, December 2007.

[10] C. Kim, M. Caesar, A. Gerber, and J. Rexford, "Revisiting route caching: The world should be flat," in Passive and Active Network Measurement, 2009, pp.3-12.

[11] L. Colitti, S.H. Gunderson, E. Kline, and T. Refice, "Evaluating IPv6 Adoption in the Internet", in Proc. passive and active measurement (PAM), 2010, pp.141-150.

[12] D. Malone, "Observations of IPv6 addresses." In Proc. passive and active network measurement (PAM), 2008, pp.21-30.

[13] E. Karpilovsky, A. Gerber, D. Pei, J. Rexford, and A. Shaikh, "Quantifying the Extent of IPv6 Deployment", in Proc. passive and active measurement (PAM), 2009, pp.13-22.

[14] P. Hicks, "Fraction of IPv6 traffic (in packets and bytes) for monthly passive traces", May 2011, http://www.caida.org/data/passive/trace_stats/ipv6_traffic.xml.

[15] L. Gao, J. Yang, H. Zhang, D. Qin and B. Zhang, "What's Going On in Chinese IPv6 World", in Proc. Network Operations and Management Symposium (NOMS), 2012, pp.534-537.

[16] F. Li, C. An, J. Yang, P. Wu and Z. Chen. "Unravel the characteristics and development of current IPv6 network", in Proc. Local Computer Networks (LCN), 2012, pp. 316-319.

[17] Internet Society World IPv6 Day, 2011, http://isoc.org/wp/worldipv6day.

[18] N. Sarrar, G. Maier, B. Ager, R. Sommer, and S. Uhlig, "Investigating IPv6 Traffic - What Happened at the World IPv6 Day?", in Proc. passive and active measurement (PAM), 2012, pp:11-20.

[19] M. Nikkhah, R. Guérin, Y. Lee, and R. Woundy, "Assessing IPv6 through web access a measurement study and its findings", in Proc. Conference on emerging Networking EXperiments and Technologies (CoNEXT), 2011, pp.26:1-26:12.

[20] A. Dhamdhere, M. Luckie, B. Huffaker, K. claffy, A. Elmokashfi and E. Aben, "Measuring the Deployment of IPv6: Topology, Routing and Performance", in Proc. Internet measurement conference (IMC), 2012, pp.537-550.

[21] K. Thompson, G. J. Miller, and R. Wilder. (1997). Wide-area Internet traffic patterns and characteristics. Network, IEEE, 11(6), pp.10-23.

[22] M. J. Akhbarizadeh and M. Nourani. Efficient prefix cache for network processors. In High Performance Interconnects, 2004. Proceedings. 12th Annual IEEE Symposium on. IEEE. pp.41-46.

[23] Z. Huang, G. Liu, and J. k. Peir. Greedy prefix cache for IP routing lookups. In Pervasive Systems, Algorithms, and Networks (ISPAN), 2009 10th International Symposium on. IEEE. pp. 92-97.

[24] Y. Liu, S. O. Amin, and L. Wang. Efficient FIB caching using minimal non-overlapping prefixes. ACM SIGCOMM Computer Communication Review, 43(1), pp.14-21.

[25] H. Liu. Reducing cache miss ratio for routing prefix cache. In Global Telecommunications Conference, 2002. GLOBECOM'02. IEEE. pp. $2323-2327$.

[26] H. Liu. Routing prefix caching in network processor design. In Computer Communications and Networks, 2001. Proceedings. Tenth International Conference on. IEEE. pp. 18-23.

[27] A. Akella, B. Maggs, S. Seshan, A. Shaikh, R. Sitaraman, "A measurement-based analysis of multihoming”, in Proc. of ACM conference on SIGCOMM, 2003, pp. 353-364.

[28] A. Akella, J. Pang, B. Maggs, S. Seshan, A. Shaikh, "A comparison of overlay routing and multihoming route control”, in Proc. of ACM conference on SIGCOMM, pp. 93-106.

[29] D. K. Goldenberg, L. Qiuy, H. Xie, Y. R. Yang, Y. Zhang, "Optimizing cost and performance for multihoming", ACM SIGCOMM Computer Communication Review, Vol. 34, No. 4, pp. 79-92.

[30] H. H. Liu, Y. Wang, Y. R. Yang, H. Wang, C. Tian, "Optimizing cost and performance for content multihoming”, in Proc. of ACM conference on SIGCOMM, 2012, pp. 371-382.

[31] J. M. Wang, J. Zhang, B. Bensaou, "Content multi-homing: an alternative approach", in Proc. of IEEE International Conference on Communications, 2014, pp. 3118-3123.

[32] C. De Launois, B. Quoitin, O. Bonaventure, "Leveraging network performance with IPv6 multihoming and multiple provider-dependent aggregatable prefixes, Computer Networks, 50(8), pp. 1145-1157.

[33] T. Benson, A. Akella, and D. Maltz, "Unraveling Complexity in Network Management”, in Proc. of USENIX NSDI, Boston, MA, Apr. 2009.

[34] T. Benson, A. Akella, and A. Shaikh, "Demystifying configuration challenges and trade-offs in network-based ISP services", in Proc. of ACM SIGCOMM. 2011, 302-313.

[35] F. Wang, Z. M. Mao, J. Wang, L. Gao, and R. Bush, "A measurement study on the impact of routing events on end-to-end Internet path performance," in Proc. ACM SIGCOMM, 2006. 
[36] S. Deshpande, M. Thottan, T.K. Ho, B. Sikdar, "An online mechanism for BGP instability detection and analysis," IEEE Transactions on Computers, vol 58(11), 2009, pp.1470-1484.

[37] F. Wang, J. Qiu, L. Gao, J. Wang, On understanding transient interdomain routing failures, IEEE/ACM Transactions on Networking, vol.17(3), 2009, pp.740-751.

[38] F. Li, C. An, J. Yang, J. Wu, H. Zhang, “A study of traffic from the perspective of a large pure ISP”, Computer Communications, 2014, Vol.37, pp.40-52.

[39] J. Wu, J. H. Wang, and J. Yang, "CNGI-CERNET2: an IPv6 Deployment in China”, ACM SIGCOMM Computer Communication Review, 2011, Vol.41, No. 2, pp: 48-52.

[40] Expect. http://expect.sourceforge.net/.

[41] Quagaa Routing Suite. http://www.nongnu.org/quagga/.

[42] Really Awesome New Cisco ConfIg Differ (RANCID). http://www.shrubbery.net/rancid/, 2004.

[43] Subversion. http://en.wikipedia.org/wiki/Subversion_(software).

[44] Z. Zhang, M. Zhang, A. G. Greenberg, Y.C. Hu, R. Mahajan, B. Christian, "Optimizing Cost and Performance in Online Service Provider Networks," in Proc. NSDI, 2010, pp. 33-48.

[45] https://www.sixxs.net/wiki/IPv6_Enabled_Hosting/.

[46] C. Labovitz, A. Ahuja, A. Bose, F. Jahanian, "Delayed Internet routing convergence,” ACM SIGCOMM Computer Communication Review, vol.30(4), pp. $175-187$.

[47] University of Oregon Route Views Project. http://route-views6.routeviews.org/.

[48] Fuliang Li, Jiahai Yang, Jianping Wu, Zhiyan Zheng, Huijing Zhang, Xingwei Wang, "Configuration Analysis and Recommendation: Case Studies in IPv6 Networks," Computer Communications, 2014, 53:37-51.

[49] A. Berger, N. Weaver, R. Beverly, and L. Campbell. "Internet nameserver IPv4 and IPv6 address relationships", in Proc. Internet Measurement Conference (IMC), Barcelona, Spain, 2013, pp. 91-104.

[50] J. Czyz, K. Lady, S.G. Miller, M. Bailey, M. Kallitsis, and M. Karir. "Understanding IPv6 internet background radiation”, in Proc. Internet Measurement Conference (IMC), Barcelona, Spain, 2013, pp. 105-118.

[51] M. Luckie, R. Beverly, and W. Brinkmeyer. "Speedtrap: internet-scale IPv6 alias resolution”, in Proc. Internet Measurement Conference (IMC), Barcelona, Spain, 2013, pp. 119-126.

[52] R. Beverly, W. Brinkmeyer, M. Luckie, and J.P. Rohrer. "IPv6 alias resolution via induced fragmentation", in Proc. Passive and Active Measurement (PAM), 2013, pp. 155-165

[53] A. Lutu, M. Bagnulo, P. Cristel, and M. Olaf. "Understanding the Reachability of IPv6 Limited Visibility Prefixes", in Proc. Passive and Active Measurement (PAM), 2014, pp. 163-172.

[54] H.A. Alzoubi, M. Rabinovich, and O. Spatscheck. "Performance implications of unilateral enabling of IPv6", in Proc. Passive and Active Measurement (PAM), 2013, pp. 115-124.

[55] J. Czyz, M. Allman, J. Zhang, S. Iekel-Johnson, E. Osterweil, M. Bailey, "Measuring IPv6 Adoption”, in Proc. of ACM conference on SIGCOMM, 2014, pp. $87-98$

[56] V. Giotsas, M. Luckie, B. Huffaker, K. Claffy, "IPv6 AS Relationships, Cliques, and Congruence”, in Proc. of Passive and Active Measurement (PAM), 2015, pp. 111-122.

[57] M. Luckie, B. Huffaker, A. Dhamdhere, V. Giotsas, “AS relationships, customer cones, and validation”, in Proc. of Internet Measurement Conference (IMC), Barcelona, Spain, 2013, pp. 243-256.

[58] R. Beverly, M. Luckie, L. Mosley, K. Claffy, "Measuring and Characterizing IPv6 Router Availability", in Proc. of Passive and Active Measurement (PAM), 2015, pp. 123-135.

[59] R. Padmanabhan, Z. Li, D. Levin, N. Spring, "UAv6: Alias Resolution in IPv6 Using Unused Addresses", in Proc. of Passive and Active Measurement (PAM), 2015, pp. 136-148.

[60] R. Beverly, A. Berger, "Server Siblings: Identifying Shared IPv4/IPv6 Infrastructure via Active Fingerprinting", in Proc. of Passive and Active Measurement (PAM), 2015, pp. 149-161.

[61] R. Guo, J. G. Delgado-Frias, "IP Routing table compaction and sampling schemes to enhance TCAM cache performance", Journal of Systems Architecture, 55(1), pp. 61-69.

[62] D. Plonka, A. Berger, "Temporal and Spatial Classification of Active IPv6 Addresses", in Proc. of Internet Measurement Conference (IMC), 2015, pp. 509522. 